\title{
RESULTS ON HEAVY QUARK SELECTION THROUGH AN IMPACT PARAMETER TRIGGER
}

\author{
M. Adamovitch, Y. Alexandrov, S. Gerasimov, S. Kharlamov, L. Malinina, M. Zavertiaev \\ Lebedev Institute of Physics, Moscow, USSR \\ F. Antinori, M. Dameri, R. Hurst, B. Osculati, L. Rossi, G. Tomasini \\ University di Genova and INFN, Genova, Italy \\ J.L. Bailly, A. Buys, F. Grard, P. Legros \\ Department of Physics, Mons, Belgium \\ A. Forino, R. Gessaroli, P. Mazzanti, A. Quareni, F. Viaggi \\ INFN and University of Bologna, Bologna, Italy \\ C. Meroni, N. Redaelli, D.Torretta \\ INFN and University of Milano, Milano, Italy \\ D. Barberis, W. Beusch, F. Bourgeois, A. Corre, M. Davenport, J.P. Dufey, B.R. French, A. Jacholkowski, \\ K. Knudson, J.C. Lassalle, F. Muller, R. Pegaitaz \\ CERN, Geneva, Switzerland
}

\begin{abstract}
: this paper describes the implementation of a trigger aimed at selecting those nuclear interactions which contain a charm particle. The trigger uses a fast emulator of the PDP-11 which calculates the Impact Parameter of the decaying charged tracks originating from the charm decay. A enrichment factor of 15 is thereby obtained.
\end{abstract}

\section{1 - INTRODUCTION}

The new trigger technique presented here aims at selecting, among numerous interactions produced by a hadronic beam on a fixed target, those which show evidence for a secondary decay close to the production vertex. This is achieved by searching, among the secondary tracks, at least one that exhibits an impact parameter (i.e. the distance between the primary vertex and the direction of the track) above a minimum value.

This "Impact Parameter Trigger" (IPT) has been developed and used by the WA82 experiment at CERN, running on the OMEGA spectrometer. The physics motivation was the collection of a large sample of heavy quark state decays produced in hadronic interactions. Using a $\pi^{-}$beam of $340 \mathrm{GeV} / \mathrm{c}$, this experiment has collected up to now (October 1989) a total of $55.10^{6}$ events selected by the IPT. The offline analysis of one part ( $8.10^{6}$ triggers) has allowed us to isolate some $750 \mathrm{D}$ meson decays $\mathrm{D}^{ \pm} \rightarrow \mathrm{K} \pi \pi, \mathrm{D}^{0} \rightarrow \mathrm{K} \pi$; $\mathrm{D}^{0} \rightarrow \mathrm{K} \pi \pi \pi$.

A report on this trigger and its first results can be found in ref. [1]. Physics results are presented in refs [2] and [3]. We present here the details on the implementation of the impact parameter trigger and on the software part of the decision logic.

\section{2 - IMPACT PARAMETER TRIGGER: PRINCIPLE AND SET-UP}

The decay products of a relativistic particle of lifetime $\tau$, issued from a primary vertex, have a mean value of the impact parameter given by $\langle d\rangle=\mathrm{c} \cdot \tau \cdot$ For $\mathrm{D}$ or $\mathrm{B}$ mesons, $<\mathrm{d}>=300 \mu \mathrm{m}$.

With this order of magnitude, a selection on impact parameter values requires the use of an accurate detector, such as a telescope of silicon microstrip planes. Accuracy of the same order is also required on the primary vertex position.

In the design of the trigger system the following sources of contamination have to be considered:

- Any trajectory deflection or secondary interaction between the beam telescope and the downstream detector, including its first plane.

- Decays of strange particles.

- Ghost tracks due to spurious combinations of measurements in the downstream detector.

The implementation of the IPT for the WA82 experiment aims at simplifying the trigger algorithm as much as possible, in order to keep the decision time within values compatible with the other dead time sources (interaction trigger and data read-out). It is also designed to reduce the effect of some of the contamination sources.

In this context, the beam and secondary tracks are measured in a plane parallel to the magnetic field of the spectrometer where they appear as straight tracks. The vertical coordinate axis $\mathrm{Z}$ is parallel to the field, the $\mathrm{X}$ axis is parallel to the 
beam. The detector planes are perpendicular to the $X$ axis and measure the $\mathrm{Z}$ coordinate. So we shall only use the $\mathrm{Z}$ component of the impact parameter.

The beam is measured with two $20 \mu \mathrm{m}$ pitch microstrip planes separated by $500 \mathrm{~mm}$ from each other, the last one being at $25 \mathrm{~mm}$ in front of the target.

It is important to remove as many measurements as possible that are due to the tracks coming from the primary

vertex: when searching for a track with impact parameter, the number of combinations is reduced and so is the time spent in checking for their validity. The probability of spurious combinations is also much smaller.

This suppression is simplified by using a thin metallic target $(1.3 \mathrm{~mm})$ together with a special geometric arrangement of the downstream microstrip detector. Figure 1 shows this set up: it is made of 3 planes of microstrips perpendicular to the $\mathrm{X}$ axis, having a pitch proportional to their distance from the target. For all the tracks coming from the primary vertex, the hit number, measured from the $\mathrm{Z}$ position of the beam, is the same in the 3 planes.

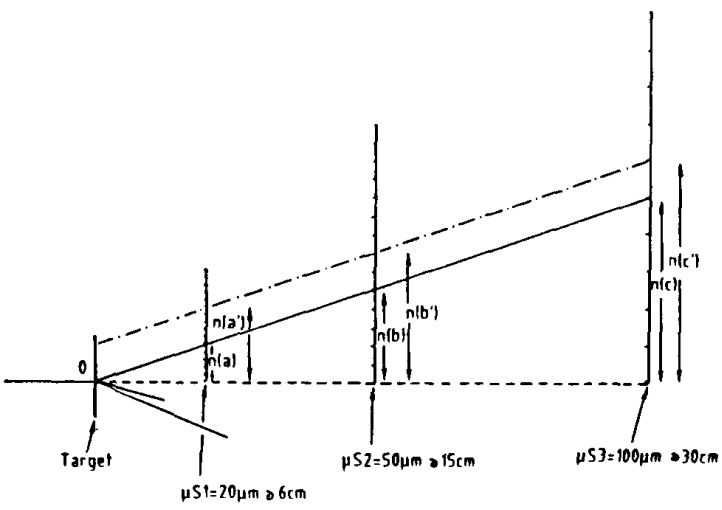

Fig. 1 - Layout of the three microstrip detectors

Conversely, these hits will have different values if they belong to a track emitted from a secondary decay, in the region between the primary vertex and the first plane.

In fact, the overlap of corresponding strips, as seen from the vertex, cannot be achieved exactly, firstly for geometrical reasons, that are related to the finite size of the strips and of the target, and secondly because of the inaccuracy in the $\mathrm{Z}$ position of the planes.The precise position of the planes is determined using beam tracks. Consequently, we have to allow for a tolerance of at most one strip when removing corresponding hits.

The planes are scanned in pairs, and the hits removed if their difference is: within 1 count, for pairs $(1,2)$ and $(1,3)$, and 0 count for $(2,3)$.
Searching for a track segment with an impact parameter value can then start. We require that this value be higher than $100 \mu \mathrm{m}$ and lower than $1000 \mu \mathrm{m}$ (in the 1989 run, the low value was $50 \mu \mathrm{m})$. To reduce the number of spurious combinations, this segment must be composed of 3 points. In addition, the contamination due to multiple scattering of low energy tracks can be reduced by requiring a confirmation of the segment by one of the OMEGA proportional chambers which is located at $2 \mathrm{~m}$ downstream of the target. The horizontal acceptance of this proportional chamber corresponds to a momentum selection of $\mathrm{p}>3.5 \mathrm{GeV} / \mathrm{c}$.

\section{3 - THE TRIGGER PROCESSOR MICE}

The impact parameter trigger algorithm is implemented in a program executed by a fast processor. For this purpose we use MICE (standing for MICroprogrammable Engine), a processor developed at CERN in 1980 [4]. It was part of the OMEGA data acquisition system, until 1988, and was used for front-end buffering and on-line filtering.

MICE has a cycle time of 105 nsec per micro-instruction. It is based on the Motorola M10800 MECL LSI high performance bit slice processor family. The standard 4 operations involved in the execution of an instruction (fetch, decode, execute, increment PC) are pipe-lined and executed simultaneously for consecutive instructions. The micro code emulates the PDP-11 MACRO assembler language; this feature allows the user to develop applications on a host, like a VAX, that provides the appropriate tools. It is possible to use the high level languages for which a suitable compiler exists. MICE is fast: for example, the association of a measurement to a plane and its normalisation takes some $2.6 \mu \mathrm{sec}$, including the loop control.

MICE has a main memory of 56 Kbytes and a micro-code storage area of 1024 words of 120 bits. The limited size of the memory is not inconvenient as long as the machine is used as a de-randomizer or a trigger element. The full data acquisition occupies $10 \mathrm{Kbytes}$ only and the size of an event is rarely exceeding 8 Kbytes.

Two interfaces are provided for data acquisition and communication with the host: one for the REMUS and RMH data acquisition systems ("read mostly" systems in use at CERN since 1975, see [5]) and the other for the connection to the host via CAMAC. The latter provides a full set of functions in order to control MICE and execute block transfers; it is implemented as a CAMAC module. Both of these interfaces can execute DMA block transfers in cycle steal mode. A set of coaxial connectors can be used to receive interrupts or to send signals to the trigger logic. All the interrupts are on the same level of priority.

With this configuration, it is possible to implement a complete front-end data acquisition system with simultaneity of the front-end read-out and the transfer to the host. In 
addition, the cycle steal mode of these transfers leaves plenty of CPU time to execute tasks such as filtering of events or software triggering.

During the 1989 run, MICE was only used as a 2nd level trigger and did not participate to the data acquisition (fig. 2). A description of the new OMEGA data acquisition system can be found in [5].

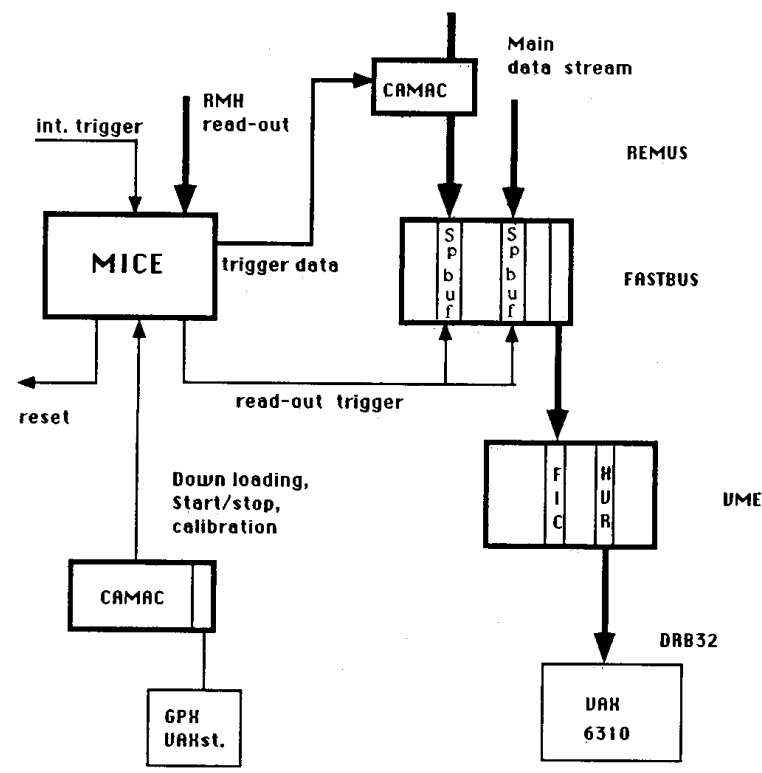

Fig. 2 - Block diagram of the data acquisition hardware

\section{4 - THE IMPACT PARAMETER PROGRAM}

\section{1 - The algorithm}

The electronic interaction trigger, that includes a multiplicity selection, ensures that the data in the microstrip planes used for the IPT fulfil the following conditions: exactly 1 hit in each of the beam planes; at least 3 hits, and at most 16 in each of the 3 downstream planes and in the proportional chamber.

On receipt of an interrupt generated by the interaction trigger, the RMH system is used to read the data from the IPT microstrip planes. The various steps of the trigger algorithm are then executed, namely:

- Decode the beam microstrip information and calculate the beam parameters.

- Decode the data for the planes downstream and normalise the hit values to the $\mathrm{Z}$ position of the interaction vertex.

- Remove corresponding hits, scanning the planes by pairs.

- Determine whether this removal was sufficient, the exact criteria being: $\mathrm{NP}_{1} \cdot \mathrm{NP}_{2} \cdot \mathrm{NP}_{3} /\left(\mathrm{N}_{1} \cdot \mathrm{N}_{2} \cdot \mathrm{N}_{3}\right)>5$, where $N P_{i}$ is the number of hits in the plane $i$ before the removal of hits and $N_{i}$ this number but after removal.
Search, among the remaining hits, for an aligned triplet of points that give an impact parameter value within the limits.

- Extrapolate this segment to the proportional chamber and search for a hit within a tolerance $(4 \times 2 \mathrm{~mm}$ pitch).

The execution stops whenever a condition rejecting the event is encountered. In such case, MICE generates a signal that resets the front-end system. These conditions are:

- A time-out during data read-out.

- An empty microstrip plane after removal of hits pointing to the interaction vertex.

- An insufficient removal of hits.

- No triplet found with an impact parameter within the specified limits.

- No confirmation of the triplet in the proportional chamber.

If the event is accepted, MICE generates a signal that starts the read-out of the whole detector. A block of data containing some information on the IPT is added.

\section{2 - The search for a triplet of points with a good impact value}

This section illustrates one aspect of the optimisation process of algorithms. One tries to avoid the combinatorial method which generates too many hypotheses that have to be checked for an impact parameter value.

In what follows, a measurement, or "hit", is the number of a micro-strip counted from the $\mathrm{Z}$ position of the interaction vertex.

With the geometrical arrangement of the planes as described before, a segment defined by a hit in each of 2 planes, say $I_{1}$ in plane 1 and $I_{3}$ in plane 3 , has an impact parameter $d$ which is proportional to the difference $I_{3}-I_{1}$ :

$$
\mathrm{d}=\mathrm{d}_{13} \cdot\left|\mathrm{I}_{3}-\mathrm{I}_{1}\right| \text {. }
$$

The factor $d_{13}$ is a constant for a given set-up and is the elementary impact increment, corresponding to a difference of one. For planes 1 and 3 it is given by:

$$
\mathrm{d}_{13}=\mathrm{p}_{3} \cdot \mathrm{X}_{1} /\left(\mathrm{X}_{3}-\mathrm{X}_{1}\right) \text {, }
$$

with $p_{3}$ being the pitch of plane $3, X_{1}$ and $X_{3}$ the position of the planes. It is assumed that : $X_{1} / p_{1}=X_{3} / p_{3}$.

Considering the measurement $I_{3}$ in plane 3 and the high cut on the impact parameter value, IPmax, one can easily determine the lowest and highest limits (hit numbers) where to search for a point in plane 1 (fig. 3 ). These values, $I_{1}$ low and $\mathrm{I}_{1}$ high, are given by:

$$
\begin{aligned}
& I_{1} \text { low }=I_{3}-I P m a x / d_{13} \\
& I_{1} \_ \text {high }=I_{3}+I P \max / d_{13}
\end{aligned}
$$

The limits for the low cut on the impact value, IPmin, are obtained by substituting IPmin to IPmax in these relations. 


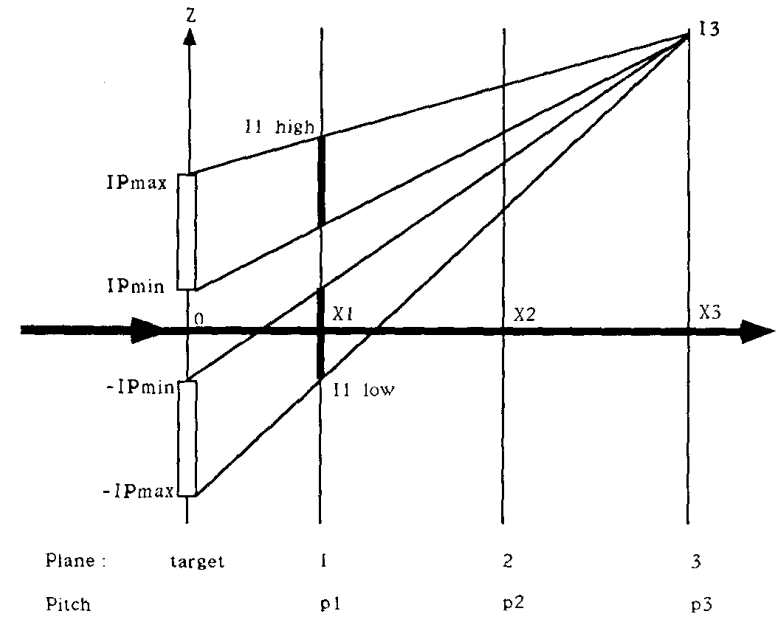

Fig. 3 - Schematic of the boundaries in the first plane

To find a triplet, the algorithm is then:

Repeat until a valid triplet is found or until all data is used:

Take next hit $\mathrm{I}_{3}$ in plane 3;

compute the limits in plane 1 ;

search for a hit $I_{1}$ in plane 1 within these limits;

if found then

compute a prediction in plane 2 for the 3rd

point (It is $1 / 4 \cdot I_{1}+3 / 4 \cdot I_{3}$ in this set-up);

search for a hit in plane 2 within a tolerance; end if;

End repeat

Note that the search for hits in planes 1 and 2 can be limited to those that are greater than the value of $I_{1}$ low calculated at the previous iteration.

Also note that the impact parameter value does not need to be calculated since the search is limited to a range of hits which gives, a priori, a valid value

\section{3 - Characteristics of the program and optimisation}

The IPT program is written in PDP MACRO-Assembler. It includes the handling of all the interrupts and signal generations, as well as the display of information at the end of burst. The trigger algorithm is coded in some 750 instructions and occupies 3.7 Kbytes, i.e. $7 \%$ of the MICE memory. It relies entirely on integer arithmetic

The computing time per event could be reduced to a minimum by carefully optimising the code at several levels:

- Optimisation of the algorithms, in order to achieve a dependency proportional to the number of hits in the planes, rather than a combinatorial dependency. Sect, 4.2 shows, as an example, the algorithm to search for a triplet having a suitable impact value.

- Optimisation of the program structure to avoid unnecessary data movements and detect the conditions of the rejects as early as possible.
- Code optimisation, such as use of the registers whenever it is possible, selection of the branching instructions taking into account their time asymmetry, and use of the special instructions provided by the emulator.

\section{4 - IPT program performance}

The real time for executing the trigger algorithm is $350 \mu \mathrm{sec}$, averaging on all types of events (accepted and rejected). The lowest and highest values are approximately $100 \mu \mathrm{sec}$ and $800 \mu \mathrm{sec}$. Adding the time to read the trigger data, the total average time is $470 \mu \mathrm{sec}$.

In the course of 3 runs (from 1987 until 1989) the total number of events selected by this program amounts to $55.10^{6}$.If one adds another $15.10^{6}$ events produced during tests and setting up runs, this total corresponds to about $10^{9}$ events submitted to the IPT selection. The program proved to be very reliable as it never crashed nor entered into a loop.

An example of the rejects due to the various selection stages is given in Table 1 . The numbers are typical of a 1 spill sample.

The total rejection factor of the IPT is approximately 8 when a minimum impact value of $50 \mu \mathrm{m}$ is required and 14 for a minimum of $100 \mu \mathrm{m}$.

TABLE 1 - Details of the rejects with a range of impact parameter value from 50 to $1000 \mu \mathrm{m}$; the values in parentheses correspond to a low cut at $100 \mu \mathrm{m}$.

\begin{tabular}{|l|l|l|l|l|}
\hline \multicolumn{1}{|c|}{ Test } & Events & Events & $\begin{array}{c}\text { \% } \\
\text { rejected } \\
\text { at this } \\
\text { stage }\end{array}$ & $\begin{array}{l}\text { \% of } \\
\text { to a } \\
\text { rejects }\end{array}$ \\
\hline $\begin{array}{l}\text { Interaction } \\
\text { trigger }\end{array}$ & 1830 & rejected & & \\
\hline $\begin{array}{l}\text { Empty plane } \\
\text { after removal } \\
\text { of hits }\end{array}$ & $1128(1123)$ & $702(707)$ & $38(39)$ & $44(41)$ \\
\hline $\begin{array}{l}\text { Sufficient } \\
\text { removal }\end{array}$ & $914 .(899)$ & $204(224)$ & $19(20)$ & $13(13)$ \\
\hline $\begin{array}{l}\text { Triplet with } \\
\text { impact }\end{array}$ & $473(243)$ & $441(656)$ & $48(73)$ & $27(38)$ \\
\hline $\begin{array}{l}\text { Confirmation } \\
\text { in propor- } \\
\text { tional chamb. }\end{array}$ & $217(105)$ & $256(138)$ & $54(57)$ & $16(8)$ \\
\hline
\end{tabular}

To determine the enrichement factor of the IPT, we have to compare the number of D's that can be extracted from a set of events having passed the IPT with the number that is obtained from a sample of events selected only by the interaction trigger. As mentioned earlier, we found 750 D's in the $8.10^{6}$ IPT events analysed so far (fig. 4a and ref [3]). A sample of $1.5 .10^{6}$ interaction triggers has been processed by the same off-line chain of programs: $9 \mathrm{D}$ events were found (fig. $4 \mathrm{~b}$ ). Taking into account the uncertainties on these numbers, one arrives at the value:

$$
\text { Enrichment factor }(100 \mu \mathrm{m})=15.6 \pm 6
$$




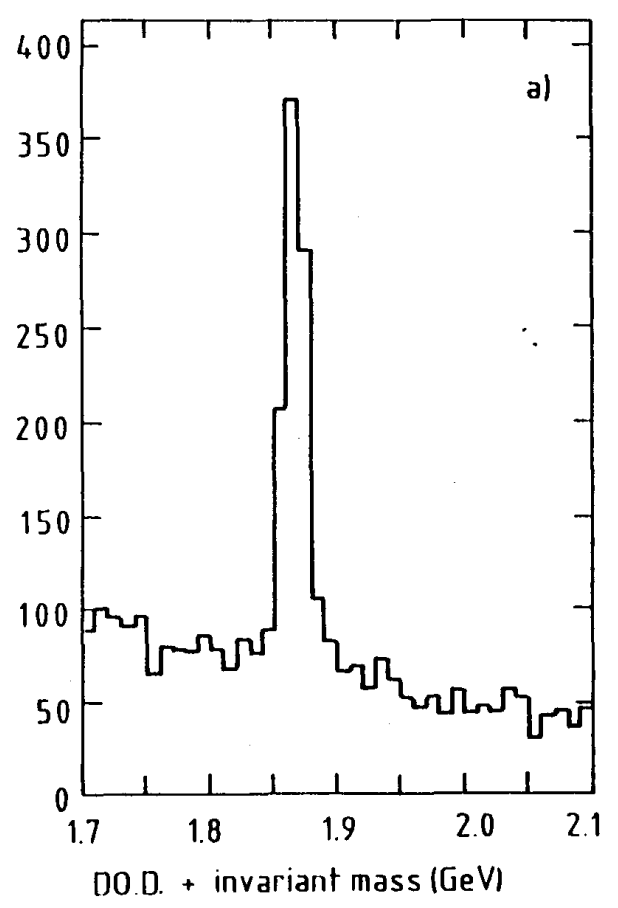

Fig. 4 - (a) D's extracted from a set of IPT events

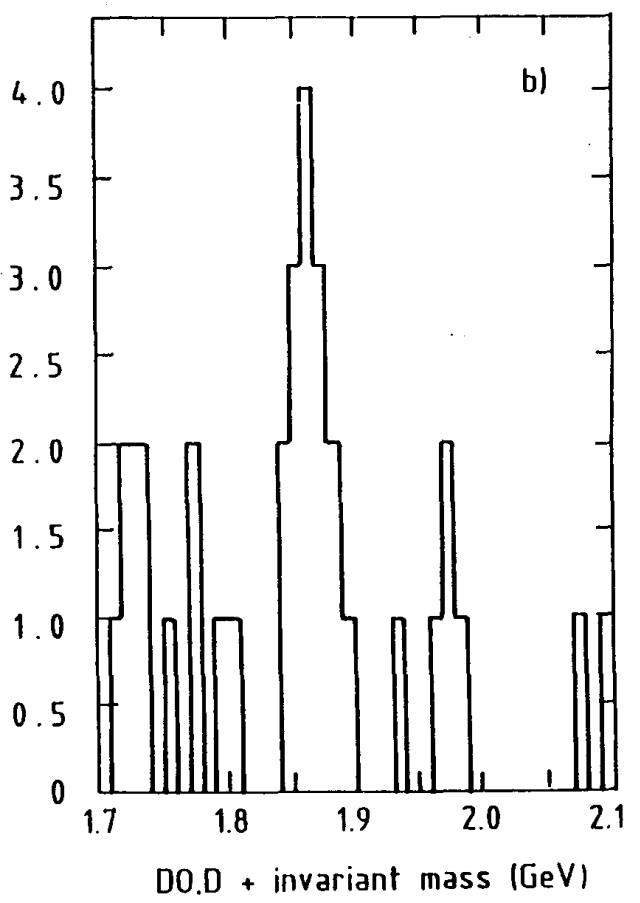

Fig. 4 - (b) D's identified in the raw data

\section{5 - PERFORMANCE OF THE OVERALL TRIGGER}

Table 2 presents typical rates at the different stages of the full trigger, as well as the dead times due to these selections.
The equivalent full dead time at the 1st interaction trigger level is $240 \mu \mathrm{sec} /$ event, thus giving a total dead time ratio of $70 \%$.

TABLE 2 - Trigger rates and dead times

\begin{tabular}{|c|c|c|}
\hline Trigger stage & $\begin{array}{l}\text { Rate/spill } \\
\text { (in } 2.6 \mathrm{sec} \text { ) }\end{array}$ & $\begin{array}{c}\text { Mean dead } \\
\text { time/event } \\
(\mu \mathrm{sec})\end{array}$ \\
\hline $\begin{array}{l}\text { Beam } \\
\text { Interaction trigger } \\
\text { Interaction trigger } \\
\text { with full dead time } \\
\text { Multiplicity } \\
\text { Impact parameter trigger } \\
\text { Full read-out }\end{array}$ & $\begin{array}{c}210^{6} \\
25000 \\
7000 \\
1830 \\
217 \\
217\end{array}$ & $\begin{array}{c}\text { Equiv: } 240 \\
58 \\
470 \\
2200\end{array}$ \\
\hline
\end{tabular}

\section{6 - CONCLUSION}

The values of the 2 factors of enrichment (15) and rejection (14), show that the IPT in its present implementation, while giving a good enrichment factor, performs also a correct rejection since it does not eliminate many good events that would have been retained by the more sophisticated selection of the off-line programs. It could of course be improved to reach a higher rejection factor.

One can envisage to improve the selectivity by searching for 2 tracks, with an impact parameter, which are crossing in the region between the target and the detector.

Another important improvement would be to reduce the IPT time which is, when combined with the trigger data readout, the main source of dead time. In future runs the reduction will be achieved by applying the technique of the contiguity processor [6].

\section{7 - REFERENCES}

[1] J.F. Baland et al., Results on the Impact Parameter Trigger for the selection of short lived particles, Nucl. Phys. B1 (1988) 303-310.

[2] M. Adamovich et al., Preliminary results on $\mathrm{D}^{ \pm}$ production properties in the interactions of $340 \mathrm{GeV} / \mathrm{c} \pi^{-}$on a target of Si and W., 24th Rencontres de Moriond, March 1988.

[3] M. Adamovich et al., Charm hadroproduction with an impact parameter trigger, International Symposium on Heavy Quark Physics, Cornell Univ., Ithaca, June 1989.

[4] J. Anthonioz-Blanc et al., MICE, a fast user programmable emulator of the PDP-11, CERNDD/80-14.

[5] F. Antinori et al., Rejuvenation of a data acquisition system for fixed target experiments in a large multiuser spectrometer at CERN. These Proceedings.

[6] G. Darbo et al., The DELPHI contiguity trigger processor, a hardware and software overview, these Proceedings. 